\title{
Probing magnetic exchange interactions with Helium
}

\author{
C. Trainer, ${ }^{1}$ C. M. Yim,,${ }^{1,2}$ C. Heil,${ }^{3}$ L. S. Farrar,${ }^{1}$ V. Tsurkan, ${ }^{4,5}$ A. Loidl,${ }^{4}$ and P. Wahl ${ }^{1}$ \\ ${ }^{1}$ SUPA, School of Physics and Astronomy, University of St Andrews, North Haugh, St Andrews, KY16 9SS, UK \\ ${ }^{2}$ Tsung Dao Lee Institute \& School of Physics and Astronomy, \\ Shanghai Jiao Tong University, Shanghai, 200240, China \\ ${ }^{3}$ Institute of Theoretical and Computational Physics, Graz University of Technology, NAWI Graz, 8010 Graz, Austria \\ ${ }^{4}$ Center for Electronic Correlations and Magnetism, Experimental Physics V, University of Augsburg, D-86159 Augsburg, Germany \\ ${ }^{5}$ Institute of Applied Physics, MD 2028 Chisinau, Republic of Moldova
}

(Dated: September 8, 2021)

\begin{abstract}
Controlling and sensing spin-polarization of electrons forms the basis of spintronics. Here, we report a study of the effect of helium on the spin-polarization of the tunneling current and magnetic contrast in spinpolarized Scanning Tunneling Microscopy. We show that the magnetic contrast in SP-STM images recorded in the presence of helium depends sensitively on the tunneling conditions. From tunneling spectra and their variation across the atomic lattice we establish that the helium can be reversibly ejected from the tunneling junction by the tunneling electrons. The energy of the tunneling electrons required to eject the helium depends on the relative spin-polarization of the tip and sample, making the microscope sensitive to the magnetic exchange interactions. We show that the time-averaged spin polarization of the tunneling current is suppressed in the presence of helium and thereby demonstrate voltage control of the spin polarization of the tunneling current across the tip-sample junction.
\end{abstract}

Spintronics is based on using the electron spin for advanced functionalities, and has been a key enabler for the increase in storage density of hard disk drives over the past decades. Tunneling magnetoresistance (TMR) is at the basis of modern hard disk read heads and magnetoresistive random access memory. While reading out magnetic information using TMR is well established, controlling spin-polarization and magnetization using electric fields is desirable for advanced spintronics devices such as spin transistors. Here, we investigate the influence of helium in a tunneling junction on the spin polarization (SP) of the tunneling current (fig. 1a), and demonstrate voltage control of the SP. The use of probe particles (PP) in a tunneling junction[1-3], such as He here, and even scanning of a contact[4-6] enables imaging with astonishing level of detail not accessible with a normal metal tip in tunneling. The PP acts as an atomic scale transducer which converts the Pauli repulsion between the orbitals of the $\mathrm{PP}$, tip and sample into changes in the tunnelling current[3]. While scanning with a magnetic adatom in contact can lead to enhanced magnetic contrast[6], the influence of PPs on the SP is less clear. In a previous study, an increased magnetic contrast of cobalt islands on $\mathrm{Pt}(111)$ [7] has subsequently been attributed to the presence of hydrogen[8]. Using a PP in spin-polarized imaging promises new control over the SP. Furthermore, in analogy to the PP providing information on the atomic forces in a nonmagnetic junction, for a magnetic junction it can be expected to reveal information on the magnetic exchange interactions.

Here we report on the effect of a PP on the SP of the tunneling current and the dependence of its binding energy on their relative magnetization. We have conducted spinpolarized scanning tunneling microscopy to determine the spin-polarization and use a probe-particle model to understand the changes in the spin-polarization of the tunneling current in the presence of helium. Tunneling spectra show clear signatures of the binding energy of the helium, which we find (a)

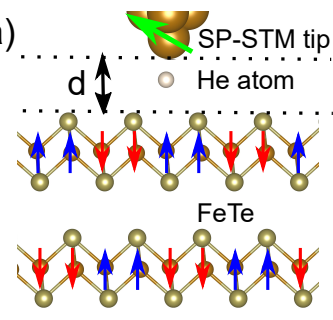

(d)
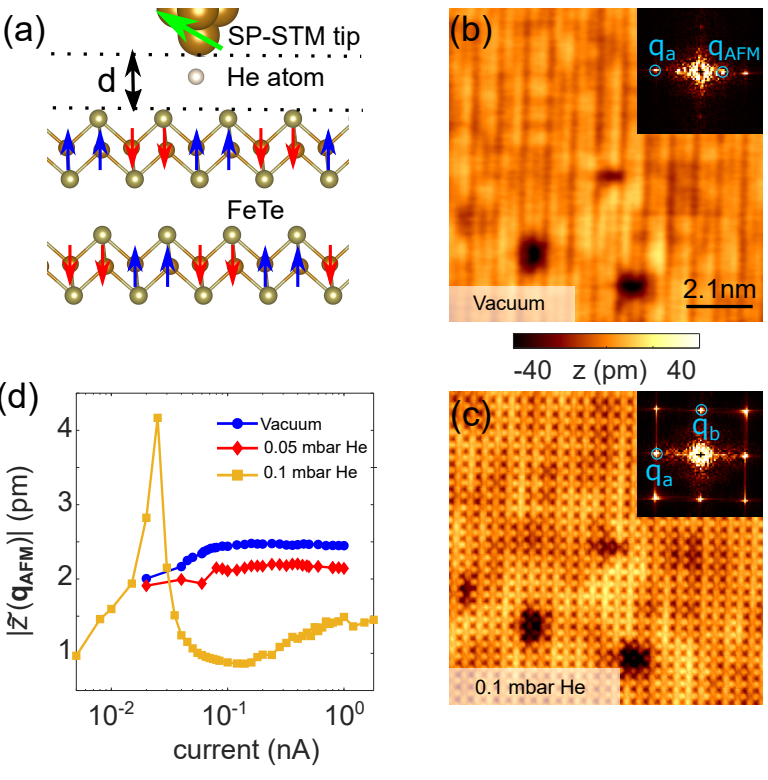

$-40 \mathrm{z}(\mathrm{pm}) \quad 40$

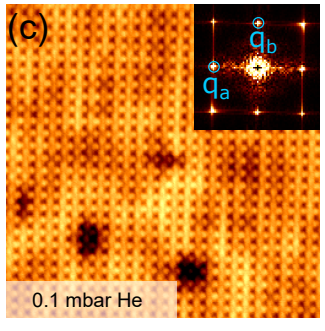

FIG. 1. (a) Schematic of the geometry of the experiment with a $\mathrm{He}$ atom as PP in the tunneling junction. $d$ denotes the tip-sample distance (see suppl. section S2A). Magnetic moments in $\mathrm{Fe}_{1+x} \mathrm{Te}$ are drawn vertical for clarity, but point into the plane of the figure in reality[9]. (b) Topographic SP-STM image $z(\mathbf{r})$ of $\mathrm{Fe}_{1+x} \mathrm{Te}$ measured in vacuum $(V=50 \mathrm{mV}, I=100 \mathrm{pA})$. The bi-collinear AFM order of $\mathrm{Fe}_{1+x} \mathrm{Te}$ is seen as a stripe-like modulation. Inset: Fourier transformation $|\tilde{z}(\mathbf{q})|$. (c) $z(\mathbf{r})$ image recorded with the same tip and in the same location as (b) after admission of He into the vacuum chamber at a partial pressure $p_{\mathrm{He}}=0.1 \mathrm{mbar}$. The magnetic contrast is hardly visible. Inset: corresponding $|\tilde{z}(\mathbf{q})|$. (d) Amplitude $\left|\tilde{z}\left(\mathbf{q}_{\mathrm{AFM}}\right)\right|$ of the Fourier peak associated with the AFM order at different tunneling currents for a bias voltage of $V=50 \mathrm{mV}$ in vacuum, and with $p_{\mathrm{He}}=0.05 \mathrm{mbar}$ and $0.1 \mathrm{mbar}$.

to be sensitive to the relative magnetization of tip and sample, 
enabling determination and imaging of the exchange interaction.

We use a low temperature STM at a temperature of $4.2 \mathrm{~K}$ to perform SP-STM measurements on a sample of iron telluride, $\mathrm{Fe}_{1.1} \mathrm{Te}$, in a $\mathrm{He}$ environment. $\mathrm{Fe}_{1+x} \mathrm{Te}$ exhibits for $x<0.12$ and at temperatures below $65 \mathrm{~K}$ a bicollinear antiferromagnetic (AFM) order [10-12] which we use to quantify the spin-polarization of the tunneling current. Samples of $\mathrm{Fe}_{1+x} \mathrm{Te}$ are cleaved in cryogenic vacuum $[13,14]$. The presence of interstitial Fe allows for in-situ preparation of magnetic tips for SP-STM [12, 15]. We have admitted controlled amounts of He into the vacuum chamber, taking care to measure only with low voltages on the scan piezo to prevent arc discharges which would destroy the STM. Bias voltages $V$ are applied to the sample with the tip at virtual ground. Differential conductance spectra $g(V)$ were recorded using a lock-in technique with a modulation voltage $V_{\text {mod }}$ (RMS). Tip-sample distances, $d$, are relative to the distance $d=0 \AA$ where the current $I(z)$ as a function of tip height $z$ changes its behaviour from exponential vacuum tunneling (see suppl. section S2A).

To model the influence of the PP on the spin-polarization of the tunneling junction, we have performed density functional theory (DFT) calculations of a surface slab of FeTe with the $\mathrm{He}$ atom and an Fe tip, as well as of surface slabs covered with a layer of $\mathrm{He}$ (for details see suppl. S1A). Tip-sample distances $d_{\mathrm{DFT}}$ in the calculations refer to the component of the distance between the top-most Te atom and the closest tip atom in the direction normal to the surface. To simulate STM tunneling currents we employ the theory of Tersoff and Hamann [16].

The surface magnetic order of $\mathrm{Fe}_{1+x} \mathrm{Te}$ is observed in topographic SP-STM images $z(\mathbf{r})$ as a stripe-like modulation along the crystallographic $a$ axis (Fig. 1b) [9, 12, 15, 17, 18]. In the Fourier transformation $\tilde{z}(\mathbf{q})$ (inset of Fig 1b), the additional modulation has a wave vector of $\mathbf{q}_{\mathrm{AFM}}=\left(\frac{1}{2}, 0\right)$, half of that of the tellurium lattice, $\mathbf{q}_{a}=(1,0)$.

Following admission of $\mathrm{He}$, the appearance of the surface changes dramatically. Fig. 1c shows a $z(\mathbf{r})$ image recorded at the same location and using the same magnetic tip and identical tunneling parameters as in Fig. 1b with a partial pressure $p_{\mathrm{He}}=0.1 \mathrm{mbar}$. The He leads to a suppression of the magnetic contrast and an enhancement in the atomic contrast. This change is also manifested in the Fourier transformation $\tilde{z}(\mathbf{q})$ (inset of Fig. 1c), where peaks associated with the magnetic order at $\mathbf{q}_{\mathrm{AFM}}$ become much weaker, while those of the crystal lattice become more intense.

In the presence of $\mathrm{He}$, the magnetic contrast becomes highly sensitive to the tunneling parameters and the partial pressure $p_{\mathrm{He}}$. Using the same magnetic tip, we have measured the intensity of the magnetic contrast $\left|\tilde{z}\left(\mathbf{q}_{\mathrm{AFM}}\right)\right|$ as a function of tunneling current $I$, and thus tip-sample distance, for fixed bias voltage $V=50 \mathrm{mV}$ in vacuum and at two different partial pressures $p_{\mathrm{He}}$. In vacuum, $\left|\tilde{z}\left(\mathbf{q}_{\mathrm{AFM}}\right)\right|$ shows only little variation with tunneling current (Fig. 1d). With $p_{\mathrm{He}}=0.05 \mathrm{mbar},\left|\tilde{z}\left(\mathbf{q}_{\mathrm{AFM}}\right)\right|$ becomes weaker, while the overall trend as a function of current remains largely the same. (a)

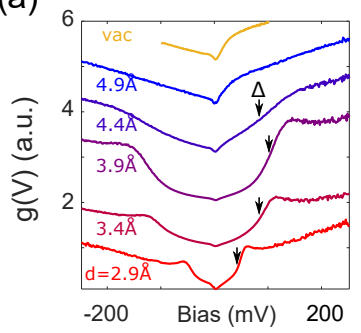

(c)

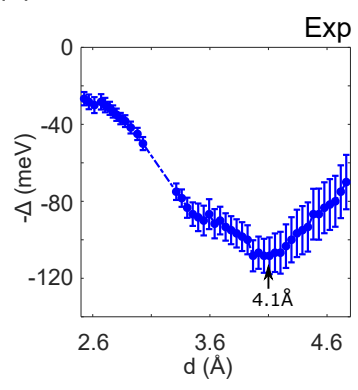

(b)

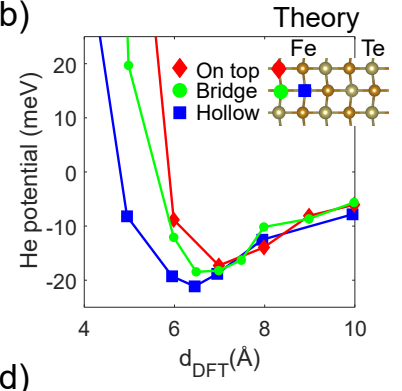

(d)

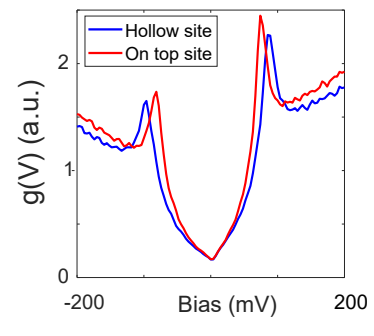

FIG. 2. Spectroscopy in He at $p_{\mathrm{He}}=0.1 \mathrm{mbar}$. (a) Differential conductance spectra $g(V)\left(V=50 \mathrm{mV}, I_{s}=80 \mathrm{pA}, V_{\text {mod }}=5 \mathrm{mV}\right)$ recorded at different $d$ in the presence of He. A $g(V)$ spectrum taken in vacuum (yellow) is also shown $\left(V=100 \mathrm{mV}, I_{s}=300 \mathrm{pA}\right.$, $V_{\text {mod }}=1 \mathrm{mV}$ ). Spectra are vertically offset for clarify and normalized at $V=250 \mathrm{mV}$. (b) DFT calculation of the trapping potential for the $\mathrm{He}$ atom in the junction for different adsorption sites. (c) The gap size $\Delta(d)$, plotted as $-\Delta(d)$, extracted from $g(V)$ spectra (defined as the inflection point at the gap edge) as a function of $d$. $\Delta(d)$ exhibits a similar functional form as the potential of the $\mathrm{He}$ atom found in the DFT calculation in (b). (d) $g(V)$ spectra recorded with the tip positioned above the hollow (blue) and on top site (red) showing a larger gap for the hollow site $(V=20 \mathrm{mV}, I=1 \mathrm{nA}$, $V_{\text {mod }}=3.5 \mathrm{mV}$ ).

At higher $p_{\mathrm{He}}=0.1 \mathrm{mbar}$, the current dependence becomes very different. $\left|\tilde{z}\left(\mathbf{q}_{\mathrm{AFM}}\right)\right|$ peaks at low current, for large tipsample distances, drops to a minimum at $I \sim 0.1 \mathrm{nA}$ before starting to rise again with increasing current and decreasing tip-sample distance. This finding suggests a significant influence of the presence of Helium on the spin-polarization of the tunneling current.

In order to understand how the presence of He affects the spin-polarization, we have characterized the junction in He by tunneling spectroscopy. Fig. 2a shows $g(V)$ spectra for different tip-sample distances $d$ at $p_{\mathrm{He}}=0.1 \mathrm{mbar}$ and a typical spectrum recorded in vacuum. The $g(V)$ spectrum recorded in vacuum is independent of $d$. When He is added, the spectrum develops a $d$-dependence and a pronounced gap-like feature whose width varies between 20 and $135 \mathrm{mV}$ for $d$ between 2.6 and $4.6 \AA$ (see suppl. fig. S6). For $d \geq 4.6 \AA$, the spectrum resembles that obtained in vacuum. The gap size is largest for $d \sim 4.1 \AA$ where the gap edge develops peak-like features.

Similar spectroscopic gaps have been observed using $\mathrm{H}_{2}$ as $\mathrm{PP}$ and were attributed to inelastic excitations of internal degrees of freedom of $\mathrm{H}_{2}$ [19], or bouncing of the $\mathrm{H}_{2}$ in the tun- 
neling junction [20]. Here, excitations due to internal degrees of freedom can be excluded as $\mathrm{He}$ is monatomic. Similarly, a vibrational mode of the He atom normal to the surface can be ruled out as the characteristic energy is expected to increase with decreasing $d$ (see suppl. section S1A), opposite to what we observe here.

We interpret the gap as the excitation gap for ejecting the He from the tunneling junction. To support this interpretation, we have modeled the trapping energy of a $\mathrm{He}$ atom in a junction consisting of an Fe tip and a FeTe surface in a DFT calculation. By changing the separation $d_{\mathrm{DFT}}$ of the tip and the sample we have mapped out the potential of the He atom in the hollow, on top and bridge sites, Fig. $2 \mathrm{~b}$. The curves resemble a Lennard-Jones potential with the preferred site being the hollow site.

We show in Fig. 2c the magnitude of the gap $\Delta$, defined as the inflection point of $g(V)$ at the gap edge, as a function of $d$ (plotted as $-\Delta(d)$ ). The qualitative shape is in good agreement with the potential energy of the He atom obtained from DFT calculations. The higher conductance once the He atom is ejected for $|V|>\Delta$ can be attributed to a larger overlap of the wave functions of tip and sample in the absence of $\mathrm{He}$ as well as contributions from inelastic processes.

Spectra acquired at the hollow and on top sites (Fig. 2d) show a difference in $\Delta$ of $10 \mathrm{mV}$ confirming this interpretation. $\Delta$ obtained from the $g(V)$ spectra is about a factor of four larger than the binding energy obtained from the DFT calculations. This difference can be explained by a lack of knowledge of the precise structure of the tip apex[21]. Measurements with different tips reproduce the $d$ dependence of $\Delta$ but with variations in shape and slight changes in the magnitude of $\Delta$ (see suppl. fig. S7.).

We can use this information about the binding energy of $\mathrm{He}$ in the different sites to model the influence of the PP on the magnetic imaging. Experimentally, we observe that the magnetic contrast $\left|\tilde{z}\left(\mathbf{q}_{\mathrm{AFM}}\right)\right|$ exhibits a strong dependence on bias voltage $V$ and $d$. As a function of $d$, the magnetic contrast $\left|\tilde{z}\left(\mathbf{q}_{\mathrm{AFM}}\right)\right|$ shows a sharp dip at $d \sim 3.7 \AA$ and a peak at $d \sim 4.5 \AA$ when imaged with $V=75 \mathrm{mV}$, whereas for larger $V$ both features become less pronounced (Fig. 3c) until the contrast becomes independent of $d$ as for vacuum tunneling (see, e.g, curve for $V=200 \mathrm{mV}$ ). In the following, we will introduce a model to explain (a) the suppression of spin-polarization for small $d$ and bias voltages $V$ and (b) the enhanced magnetic contrast at larger $d$.

While in our experiment we expect the PP to follow the STM tip, we have simulated STM images to approximate the imaging process in a slab calculation with $\mathrm{He}$ atoms placed at different adsorption sites on the surface. These calculations reproduce similar contrast as observed in the experimental images though with no significant suppression of the magnetic contrast. Fig. 3a shows a simulated image for $\mathrm{He}$ in the hollow site, showing excellent agreement with the image obtained from experiment at $d=4.1 \AA$. SP images are obtained from the spin-resolved densities of states $\rho_{\uparrow}(\mathbf{r})$ and $\rho_{\downarrow}(\mathbf{r})$ to
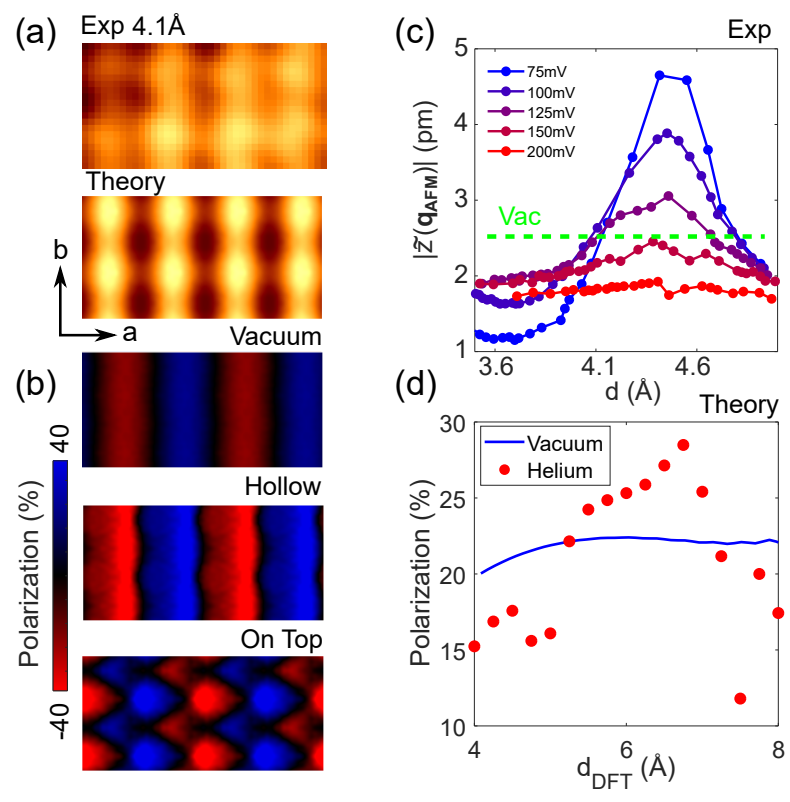

FIG. 3. (a) $z(\mathbf{r})$ image for $d=4.1 \AA(V=50 \mathrm{mV}, I=30 \mathrm{pA})$ and the simulated topography for $d_{\mathrm{DFT}}=7.6 \AA$ for comparison. (b) calculated SP at the same $d_{\mathrm{DFT}}$ for vacuum tunnling and for a layer of $\mathrm{He}$ in the hollow and the on top sites. The largest magnetic contrast is observed for He in the hollow site. (c) Amplitude $\left|\tilde{z}\left(\mathbf{q}_{\mathrm{AFM}}\right)\right|$ of the magnetic order as a function of $d$ and for a range of voltages $V$. The green dashed line represents the value for vacuum tunneling. (d) The SP $P$ extracted from the amplitude of the magnetic order in the simulated images. Red points are extracted from our model accounting for the He atom probing different sites during the measurement (for details see suppl. S1B). The blue curve is the intensity of the magnetic order for a vacuum junction.

determine $P=\frac{\rho_{\uparrow}-\rho_{\downarrow}}{\rho_{\uparrow}+\rho_{\downarrow}}$ at a height $d_{\mathrm{DFT}}$ above the surface (fig. 3b). From the calculations and with a He atom in the hollow site, we find an overall increase in the spin-polarization of up to $\sim 30 \%$ compared to without $\mathrm{He}$, while He in the on-top position leads to a more complex pattern. We attribute these results to the effect of Pauli repulsion reducing the density of states above the He atom (see suppl. S1A). When the He is at a Te site it suppresses tunneling to the Te $p$ orbitals more than tunnelling to the spin-polarized $\mathrm{Fe} d$ states. This results in a phase shift of the magnetic order in the simulated images dependent on the adsorption site of the $\mathrm{He}$ (see fig. $3 \mathrm{~b}$ and suppl. fig S2). The suppression we observe experimentally suggests that the He atom does not stay in the same site during imaging, but probes a distribution of sites closest to the tip. To model this imaging process we account for the potential energy landscape of the $\mathrm{He}$ atom in the presence of the tip, using the calculated potential energy curves (fig.2b). We estimate the binding energy of the $\mathrm{He}$ atom at each site for a given tip position and then combine the calculated SP images weighting their contributions using a Boltzmann factor. The resultant behaviour of the SP as a function of $d_{\mathrm{DFT}}$ obtained from the calculation (Fig. 3d) reproduces the suppression in 
(a)

(b)
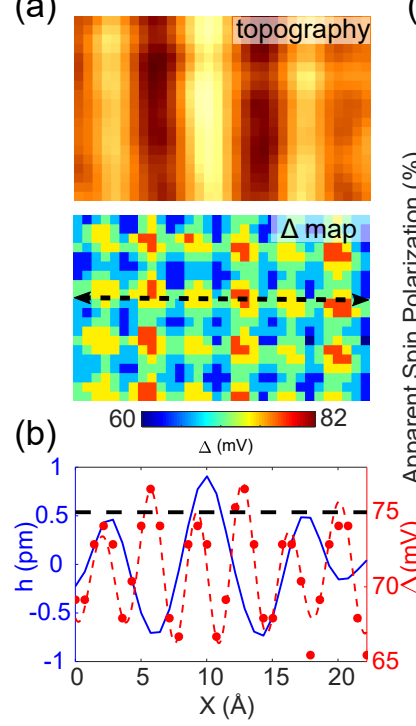

(c)
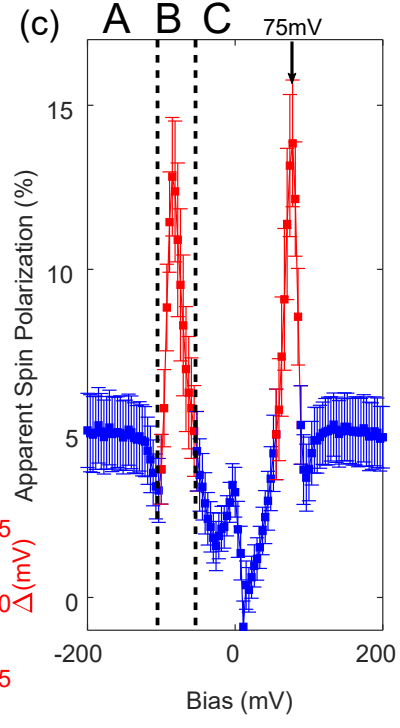

FIG. 4. (a) Topography $z(\mathbf{r})(V=200 \mathrm{mV}, I=1 \mathrm{nA})$ and spatial map of binding energy $\Delta(\mathbf{r})$ of the He atom extracted from a spectroscopic map $g(\mathbf{r}, V)\left(V=20 \mathrm{mV}, I=1 \mathrm{nA}, V_{\text {mod }}=3.5 \mathrm{mV}\right)$ acquired with a setpoint at which the magnetic contrast is not visible in the topography. The $\Delta(\mathbf{r})$ map shows clear variations between different atomic sites. (b) average profile from $z(\mathbf{r})$ (blue curve) and along the dashed line from $\Delta(\mathbf{r})$ (red curve) in a. Besides the difference in $\Delta$ between the hollow and on top sites, $\mathrm{a} \sim 3 \mathrm{mV}$ modulation is observed between hollow sites with different relative magnetization of tip and sample. (c) The apparent SP of the tunnel junction extracted from the $g(\mathbf{r}, V)$ map. Blue points show true spin polarization, red points show enhancement due to the exchange interaction, leading to a giant apparent SP. There are three regimes: A - He is ejected from the junction, $\mathrm{B}-\mathrm{He}$ is trapped only at some sites and for one relative magnetic configuration of tip and sample, and $\mathrm{C}-\mathrm{He}$ remains always in the junction.

SP seen experimentally for small $d$ (fig. 3c) and also exhibits an increase for larger $d$ when the He atom is trapped mostly in the hollow site. The change in preferred adsorption site is accompanied by a phase shift which is also seen experimentally (see fig. S3).

We conclude that the suppression of spin-polarization for small $d$ is due to the He atom hopping between neighbouring sites, scrambling the information of the spin-polarization over the time of the measurement. However, the increase in SP seen at some values of $d$ obtained from the model is significantly lower compared to that seen experimentally: in the experiment, we observe an increase in magnetic contrast by almost a factor of two, whereas the calculation can only account for an increase by about $33 \%$ compared to vacuum tunneling. Therefore, the enhanced magnetic contrast requires a different mechanism.

To better understand the strong increase in magnetic contrast at $d \approx 4.5 \AA$ and $V=75 \mathrm{mV}$ we spatially map the binding energy $\Delta$ at the atomic scale from a spectroscopic map $g(\mathbf{r}, V)$. In a $\Delta(\mathbf{r})$ map (Fig. 4a) extracted from the $g(\mathbf{r}, V)$ map with $\mathrm{He}$ in the tunneling junction, one can clearly see the variation of $\Delta$ between the on-top, hollow and bridge sites.

The map also reveals that the binding energy of the $\mathrm{He}$ atom depends on the relative orientation of the tip and sample magnetization. Fig. $4 \mathrm{~b}$ shows that $\Delta$ varies between otherwise equivalent hollow sites with parallel and antiparallel orientation of the magnetizations of tip and sample by about $3 \mathrm{meV}$. The binding energy of the $\mathrm{He}$ is thus sensitive to the exchange interaction between the tip and the sample, providing a new way to image the magnetic order of the surface: By adjusting the bias voltage to between the energies which eject the $\mathrm{He}$ from the two hollow sites with opposite magnetization, here about $75 \mathrm{mV}$, the He is ejected for one relative configuration, but not the other leading to a huge amplification in the magnetic contrast.

We can confirm this picture by extracting the apparent SP from the $g(\mathbf{r}, V)$ map (fig. 4c), $P(V)=\frac{g_{\uparrow \uparrow}(V)-g_{\uparrow \downarrow}(V)}{g_{\uparrow \uparrow}(V)+g_{\uparrow \downarrow}(V)}=$ $\frac{\left|\tilde{g}\left(V, \mathbf{q}_{A F M}\right)\right|}{\tilde{g}(V, \mathbf{q}=0)}[22,23]$. For $|V| \gg \Delta$ (region A) the He atom is ejected from the junction and the SP is comparable to that seen in vacuum tunneling. A huge apparent enhancement of the SP is found for $|V| \sim \Delta$ (region B) when the difference in binding energy of He for different relative magnetisation of tip and sample straddles the bias voltage $V$, leading to $\mathrm{He}$ being ejected for one relative configuration, but not for the other. For $|V| \ll \Delta$, there is a strong suppression of the SP (region $\mathrm{C}$ ) due to the presence of $\mathrm{He}$.

The suppression we observe in region $\mathrm{C}$ also explains why in all but one [24] of the previous studies of FeTe by STM, signatures of the bi-collinear magnetic order have been observed $[12,15,17,18,25]$. The experiment in ref. 24 was conducted in He, likely explaining why no magnetic order was observed.

We show that the time-averaged spin-polarization of the tunneling current can be suppressed by the presence of helium in the junction due to the dynamics of the probe particle. Using the bias voltage, the PP can be ejected in a controlled way from the tunneling junction, thus enabling control of the average spin polarization of the current. We further demonstrate that the probe particle can be used to determine exchange interactions between the tip and sample in a scanning tunneling microscope, complementing direct force measurements[26].

We gratefully acknowledge useful discussions with Pavel Jelinek. CT and PW acknowledge support from EPSRC (EP/R031924/1) and CMY and LSF from EP/S005005/1. C.H. acknowledges support by the Austrian Science Fund (FWF) Project No. P32144-N36 and the VSC-4 of the Vienna University of Technology. The work was partially supported by the Deutsche Forschungsgemeinschaft (DFG) through Transregional Research Collaboration TRR 80 (Augsburg, Munich, and Stuttgart).

[1] C. Wagner and R. Temirov, Prog. Surf. Sci. 90, 194 (2015).

[2] R. Temirov, S. Soubatch, O. Neucheva, A. C. Lassise, and F. S. Tautz, New J. Phys. 10, 053012 (2008). 
[3] C. Weiss, C. Wagner, C. Kleimann, M. Rohlfing, F. S. Tautz, and R. Temirov, Phys. Rev. Lett. 105, 086103 (2010).

[4] Y.-h. Zhang, P. Wahl, and K. Kern, Nano Lett. 11, 3838 (2011).

[5] Y.-h. Zhang, P. Wahl, and K. Kern, Phys. Rev. B 87, 205417 (2013).

[6] S. Ouazi, A. Kubetzka, K. von Bergmann, and R. Wiesendanger, Phys. Rev. Lett. 112, 076102 (2014).

[7] S. Rusponi, N. Weiss, T. Cren, M. Epple, and H. Brune, Appl. Phys. Lett. 87, 162514 (2005).

[8] W. A. Hofer, K. Palotás, S. Rusponi, T. Cren, and H. Brune, Phys. Rev. Lett. 100, 026806 (2008).

[9] C. Trainer, M. Songvilay, N. Qureshi, A. Stunault, C. M. Yim, E. E. Rodriguez, C. Heil, V. Tsurkan, M. A. Green, A. Loidl, P. Wahl, and C. Stock, Phys. Rev. B 103, 024406 (2021).

[10] C. Koz, S. Rößler, A. A. Tsirlin, S. Wirth, and U. Schwarz, Phys. Rev. B 88, 094509 (2013).

[11] W. Bao, Y. Qiu, Q. Huang, M. A. Green, P. Zajdel, M. R. Fitzsimmons, M. Zhernenkov, S. Chang, M. Fang, B. Qian, E. K. Vehstedt, J. Yang, H. M. Pham, L. Spinu, and Z. Q. Mao, Phys. Rev. Lett. 102, 247001 (2009).

[12] M. Enayat, Z. Sun, U. R. Singh, R. Aluru, S. Schmaus, A. Yaresko, Y. Liu, C. Lin, V. Tsurkan, A. Loidl, J. Deisenhofer, and P. Wahl, Science 345, 653 (2014), https://science.sciencemag.org/content/345/6197/653.full.pdf.

[13] S. C. White, U. R. Singh, and P. Wahl, Rev. Sci. Instrum. 82, 113708 (2011).

[14] C. Trainer, C. M. Yim, M. McLaren, and P. Wahl, Rev. Sci.
Instrum. 88, 093705 (2017).

[15] U. R. Singh, R. Aluru, Y. Liu, C. Lin, and P. Wahl, Phys. Rev. B 91, 161111(R) (2015).

[16] J. Tersoff and D. R. Hamann, Phys. Rev. B 31, 805 (1985).

[17] T. Hänke, U. R. Singh, L. Cornils, S. Manna, A. Kamlapure, M. Bremholm, E. M. J. Hedegaard, B. B. Iversen, P. Hofmann, J. Hu, Z. Mao, J. Wiebe, and R. Wiesendanger, Nat. Commun. 8, 13939 (2017).

[18] C. Trainer, C. M. Yim, C. Heil, F. Giustino, D. Croitori, V. Tsurkan, A. Loidl, E. E. Rodriguez, C. Stock, and P. Wahl, Sci. Adv. 5, eaav3478 (2019).

[19] J. A. Gupta, C. P. Lutz, A. J. Heinrich, and D. M. Eigler, Phys. Rev. B 71, 115416 (2005).

[20] S. Li, A. Yu, F. Toledo, Z. Han, H. Wang, H. Y. He, R. Wu, and W. Ho, Phys. Rev. Lett. 111, 146102 (2013).

[21] X. Bouju, C. Girard, H. Tang, C. Joachim, and L. Pizzagalli, Phys. Rev. B 55, 16498 (1997).

[22] M. Bode, Rep. Prog. Phys 66, 523 (2003).

[23] R. Wiesendanger, Rev. Mod. Phys. 81, 1495 (2009).

[24] T. Machida, K. Kogure, T. Kato, H. Nakamura, H. Takeya, T. Mochiku, S. Ooi, Y. Mizuguchi, Y. Takano, K. Hirata, and H. Sakata, J. Phys. Soc. Japan 81, 074714 (2012), https://doi.org/10.1143/JPSJ.81.074714.

[25] W. Li, W.-G. Yin, L. Wang, K. He, X. Ma, Q.-K. Xue, and X. Chen, Phys. Rev. B 93, 041101 (2016).

[26] U. Kaiser, A. Schwarz, and R. Wiesendanger, Nature 446, 522 (2007). 